\title{
ON A FAMILY OF INTEGRAL OPERATORS RELATED TO FRACTIONAL CALCULUS
}

\author{
By YÛSAKU KOMATU
}

\section{Introduction.}

Let $I$ denote the class of analytic functions $f$ regular in the unit disk $E$ $=\{|z|<1\}$ and normalized at the origin by $f(0)=0$ and $f^{\prime}(0)=1$. On the other hand, let $\sigma$ be a probability measure supported by the unit interval $I=[0,1]$. Then the linear integral operator $\mathcal{L}$ is defined on $\mathscr{I}$ by the expression

$$
\mathcal{L} f(z)=\int_{I} \frac{f(z t)}{t} d \sigma(t) .
$$

It is readily seen that $f \in \mathscr{F}$ implies $\mathcal{L} f \in \mathscr{F}$.

Let the Taylor expansion of $f \in \mathscr{F}$ be given by

$$
f(z)=\sum_{\nu=1}^{\infty} c_{\nu} z^{\nu} \quad \text { with } c_{1}=1 .
$$

Then substitution followed by termwise integration yields

$$
\mathcal{L} f(z)=\sum_{\nu=1}^{\infty} \alpha_{\nu} c_{\nu} z^{\nu}
$$

where $\left\{\alpha_{\nu}\right\}_{\nu=1}^{\infty}$ is the moment sequence with respect to $\sigma$ defined by

$$
\alpha_{\nu}=\int_{I} t^{\nu-1} d \sigma(t) \quad(\nu=1,2, \cdots),
$$

which is decreasing and nonnegative; in particular, $\alpha_{1}=1$.

The iteration $\left\{\mathcal{L}^{n}\right\}_{n=0}^{\infty}$ arises automatically by $\mathcal{L}^{0}=\mathrm{id}, \mathcal{L}^{n}=\mathcal{L} \mathcal{L}^{n-1}(n=1$, $2, \cdots)$ or by

$$
\mathcal{L}^{n} f(z)=\sum_{\nu=1}^{\infty} \alpha_{\nu}{ }^{n} c_{\nu} z^{\nu}
$$

We discussed in $[2,3]$ the problem of interpolating the sequence $\left\{\mathcal{L}^{n}\right\}$ into a family $\left\{\mathcal{L}^{\lambda}\right\}$ depending on a continuous parameter $\lambda$ in such a way that the additivity $\mathcal{L}^{\lambda} \mathcal{L}^{\mu}=\mathcal{L}^{\lambda+\mu}$ remains valid. We then derived several properties of the family thus introduced, and observed the simplest distinguished case gener.

Received September 29, 1986 
ated by a special probability measure $\sigma(t)=t$ especially in detail.

In the present paper we shall mainly observe the case generated by the probability measure involving a real parameter $a$ defined by

$$
\sigma(t ; a)=t^{a} \quad \text { with } a>0 .
$$

The measure $\sigma(t ; 1)=t$ is indeed included as a particular one but it plays an exceptional role occasionally in certain sense.

\section{Family generated by $t^{a}$.}

We now suppose that a probability measure $\sigma$ possesses the density $\rho$ :

$$
\sigma(t)=\int_{0}^{t} \rho(\tau) d \tau ; \quad \rho(\tau) \geqq 0, \quad \int_{I} \rho(\tau) d \tau=1 .
$$

The operator generated by this measure will be denoted by $\mathcal{L}[\rho]$ :

$$
\mathcal{L}[\rho] f(z)=\int_{I} \frac{f(z t)}{t} \rho(t) d t .
$$

We begin with the following lemma:

LEMMA 1. The product of two operators becomes $\mathcal{L}[p] \mathcal{L}[q]=\mathcal{L}[\rho]$ where

$$
\rho(t)=\int_{t}^{1} p(s) q\left(\frac{t}{s}\right) \frac{d s}{s} .
$$

Proof. Direct calculation shows that

$$
\begin{aligned}
\mathcal{L}[p] \mathcal{L}[q] f(z) & =\int_{I} p(s) \frac{d s}{s} \int_{I} f(z s \tau) q(\tau) \frac{d \tau}{\tau} \\
& =\int_{I} p(s) \frac{d s}{s} \int_{0}^{s} f(z t) q\left(\frac{t}{s}\right) \frac{d t}{t}=\int_{I} f(z t) \frac{d t}{t} \int_{t}^{1} p(s) q\left(\frac{t}{s}\right) \frac{d s}{s} \\
& =\int_{I} \frac{f(z t)}{t} \rho(t) d t=\mathcal{L}[\rho] f(z)
\end{aligned}
$$

with $\rho$ stated in the lemma.

REMARK. If we put $t=e^{-v}$ and accordingly $p(t)=P(v), q(t)=Q(v)$ and $\rho(t)$ $=R(v)$, then the expression in the lemma becomes

$$
R(v)=\int_{0}^{v} P(u) Q(v-u) d u .
$$

This shows that $R$ is the convolution of $P$ and $Q: R=P * Q$.

LEMMA 2. Let every member $\mathcal{L}^{\lambda}$ of the family $\left\{\mathcal{L}^{\lambda}\right\}_{\lambda>0}$ be generated by a 
measure with the density $\rho_{\lambda}: \mathcal{L}^{\lambda}=\mathcal{L}\left[\rho_{\lambda}\right]$. Then the additivity $\mathcal{L}^{\lambda} \mathcal{L}^{\mu}=\mathcal{L}^{\lambda+\mu}$ is characterized by

$$
\int_{t}^{1} \rho_{\lambda}(s) \rho_{\mu}\left(\frac{t}{s}\right) \frac{d s}{s}=\rho_{\lambda+\mu}(t)
$$

Proof. In view of Lemma 1 we have $\mathcal{L}\left[\rho_{\lambda}\right] \mathcal{L}\left[\rho_{\mu}\right]=\mathcal{L}[\rho]$ with

$$
\rho(t)=\int_{t}^{1} \rho_{\lambda}(s) \rho_{\mu}\left(\frac{t}{s}\right) \frac{d s}{s} .
$$

Hence the additivity is characterized by the condition that $\mathcal{L}[\rho] f(z)=\mathcal{L}\left[\rho_{\lambda+\mu}\right] f(z)$ holds for any $f \in \mathscr{F}$. This condition applied, for instance, to $f(z)=z(1-z)^{-1} \in \mathscr{T}$ yields, by comparing the coefficients of $z^{\nu}$,

$$
\int_{I} t^{\nu-1} \rho(t) d t=\int_{I} t^{\nu-1} \rho_{\lambda+\mu}(t) d t
$$

In view of the unicity of the solution of moment problem, we obtain $\rho=\rho_{\lambda+\mu}$. Conversely, if $\rho=\rho_{\lambda+\mu}$, it is evident that the additivity holds.

Now, we observe the probability measure defined by $\sigma(t ; a)=t^{a}$ with $a>0$.

THEOREM 1. The additive family of operators generated by $\sigma(t ; a)=t^{a}$ with $a>0$ is given by the probability measure $\sigma_{\lambda}(t ; a)$ with the density $\rho_{\lambda}(t ; a)$ defined by

$$
\sigma_{\lambda}(t ; a)=\int_{0}^{t} \rho_{\lambda}(\tau ; a) d \tau, \quad \rho_{\lambda}(t ; a)=\frac{a^{\lambda}}{\Gamma(\lambda)} t^{a-1}\left(\log \frac{1}{t}\right)^{\lambda-1}
$$

Proof. The condition stated in Lemma 2 can be verified by direct calculation. In fact, we have

$$
\begin{aligned}
& \int_{t}^{1} \rho_{\lambda}(s ; a) \rho_{\mu}\left(\frac{t}{s} ; a\right) \frac{d s}{s} \\
= & \frac{a^{\lambda+\mu}}{\Gamma(\lambda) \Gamma(\mu)} \int_{t}^{1} s^{a-1}\left(\log \frac{1}{s}\right)^{\lambda-1}\left(\frac{t}{s}\right)^{a-1}\left(\log \frac{s}{t}\right)^{\mu-1} \frac{d s}{s} \\
= & \frac{a^{\lambda+\mu}}{\Gamma(\lambda) \Gamma(\mu)} t^{a-1} \int_{t}^{1}\left(\log \frac{1}{s}\right)^{\lambda-1}\left(\log \frac{s}{t}\right)^{\mu-1} \frac{d s}{s} \\
= & \frac{a^{\lambda+\mu}}{\Gamma(\lambda) \Gamma(\mu)} t^{a-1}\left(\log \frac{1}{t}\right)^{\lambda+\mu-1} \int_{0}^{1} u^{\lambda-1}(1-u)^{\mu-1} d u \quad \quad\left[\log \frac{1}{s}=u \log \frac{1}{t}\right] \\
= & \frac{a^{\lambda+\mu}}{\Gamma(\lambda) \Gamma(\mu)} t^{a-1}\left(\log \frac{1}{t}\right)^{\lambda+\mu-1} \cdot \frac{\Gamma(\lambda) \Gamma(\mu)}{\Gamma(\lambda+\mu)}=\rho_{\lambda+\mu}(t ; a) .
\end{aligned}
$$

The assertion may be proved alternatively as follows. In fact, since the moment with respect to $\sigma(t ; a)$ is equal to 


$$
\alpha_{\nu}(a)=\int_{I} t^{\nu-1} d \sigma(t ; a)=\frac{a}{\nu+a-1},
$$

it is sufficient to show that the moment with respect to the measure $\sigma_{\lambda}(t ; a)$ stated in the theorem is equal to $\alpha_{\nu}(a)^{\lambda}$, what is an immediate consequence of a familiar formula

$$
\int_{I} t^{\kappa-1}\left(\log \frac{1}{t}\right)^{\lambda-1} d t=\frac{\Gamma(\lambda)}{\kappa^{\lambda}}
$$

According to Theorem 1 we shall denote in the following lines $\mathcal{L}\left[\rho_{\lambda}(t ; a)\right]$ briefly by $\mathcal{L}(a)^{\lambda}$ :

$$
\mathcal{L}(a)^{\lambda} f(z)=\frac{a^{\lambda}}{\Gamma(\lambda)} \int_{I} f(z t) t^{a-2}\left(\log \frac{1}{t}\right)^{\lambda-1} d t .
$$

The behaviors of the general family $\left\{\mathcal{L}^{\lambda}\right\}$ as $\lambda \rightarrow+0$ and $\lambda \rightarrow \infty$ have been shown in [2]. But, in case of $\sigma(t ; a)$, since the extreme exceptional cases do not appear, we can state the following theorem:

THEOREM 2. The limit relations

$$
\lim _{\lambda \rightarrow+0} \mathcal{L}(a)^{\lambda} f(z)=f(z) \quad \text { and } \quad \lim _{\lambda \rightarrow \infty} \mathcal{L}(a)^{\lambda} f(z)=z
$$

hold for every $f \in \mathscr{F}$ in $E$ uniformly in the wider sense.

On the other hand, the behaviors as $a \rightarrow+0$ and $a \rightarrow \infty$ become as follows:

THEOREM 3. The limit relations

$$
\lim _{a \rightarrow+0} \mathcal{L}(a)^{\lambda} f(z)=z \quad \text { and } \quad \lim _{a \rightarrow \infty} \mathcal{L}(a)^{\lambda} f(z)=f(z)
$$

hold for every $f \in \mathscr{F}$ in $E$ uniformly in the wider sense.

Proof. Let $z$ be restricted on any fixed compact in $E$. Then both $|f(z t) / t-z|$ and $|f(z t) / t-f(z)|$ possess for every $t \in I$ a bound $M$, say. First, we have

$$
\mathcal{L}(a)^{\lambda} f(z)-z=\frac{a^{\lambda}}{\Gamma(\lambda)} \int_{I}\left(\frac{f(z t)}{t}-z\right) t^{a-1}\left(\log \frac{1}{t}\right)^{\lambda-1} d t .
$$

For any $\varepsilon>0$ there exists $\tau \in(0,1)$ such that $|f(z t) / t-z|<\varepsilon / 2$ as $0 \leqq t<\tau$, and hence for $a<1$

$$
\left|\mathcal{L}(a)^{\lambda} f(z)-z\right|<\frac{\varepsilon}{2} \frac{a^{\lambda}}{\Gamma(\lambda)} \int_{0}^{\tau} t^{a-1}\left(\log \frac{1}{t}\right)^{\lambda-1} d t+M \tau^{a-1} \frac{a^{\lambda}}{\Gamma(\lambda)} \int_{\tau}^{1}\left(\log \frac{1}{t}\right)^{\lambda-1} d t .
$$

The first summand of this estimate is always less than $\varepsilon / 2$, while the second summand becomes less than $\varepsilon / 2$ provided $a$ is sufficiently near to zero. This 
leads to the first relation in the theorem. Next, we have

$$
\mathcal{L}(a)^{\lambda} f(z)-f(z)=\frac{a^{\lambda}}{\Gamma(\lambda)} \int_{I}\left(\frac{f(z t)}{t}-f(z)\right) t^{a-1}\left(\log \frac{1}{t}\right)^{\lambda-1} d t .
$$

For any $\varepsilon>0$ there exists $\tau \in(0,1)$ such that $|f(z t) / t-f(z)|<\varepsilon / 2$ as $1-\tau<t \leqq 1$, and hence for $a>1$

$$
\begin{aligned}
\left|\mathcal{L}(a)^{\lambda} f(z)-f(z)\right|< & M(1-\tau)^{a-1} \frac{a^{\lambda}}{\Gamma(\lambda)} \int_{0}^{1-\tau}\left(\log \frac{1}{t}\right)^{\lambda-1} d t \\
& +\frac{\varepsilon}{2} \frac{a^{\lambda}}{\Gamma(\lambda)} \int_{1-\tau}^{1} t^{a-1}\left(\log \frac{1}{t}\right)^{\lambda-1} d t
\end{aligned}
$$

Since the second summand of this estimate is always less than $\varepsilon / 2$ and the first summand becomes less than $\varepsilon / 2$ for $a$ large enough, the second relation follows.

Though the proof given here has been based on the integral representation for $\mathcal{L}(a)^{\lambda} f(z)$, a rather brief proof may be given by referring to its series expansion.

\section{Relation to integration operator.}

We have pointed out in [2] that the operator $\mathcal{L}$ with general $\sigma$ and the differentiation with respect to $\log z$ are commutative. Further, in particular case generated by $\sigma(t)=\sigma(t, 1)$, the operation $\mathcal{L}(1)^{\lambda}$ can be represented in the form

$$
\mathcal{L}(1)^{\lambda} f(z)=\frac{1}{\Gamma(\lambda)} \int_{\infty}^{\log z} f(\zeta)(\log z-\log \zeta)^{\lambda-1} d \log \zeta .
$$

Here the integration is taken along the half straight line on the log $\zeta$-plane which is parallel to the real axis and contained in the left half-plane $\{\operatorname{Re} \log \zeta$ $<0\}$. Thus, this operator coincides with the fractional integration of order $\lambda$ with respect to $\log z$. In particular, $\mathcal{L}(1)$ is just the inverse operator of $d / d \log z$.

The last-mentioned fact is peculiar to the case $a=1$. The corresponding property in the case $a \neq 1$ is stated as in the following theorem.

THEOREM 4. The operator $\mathcal{L}(a)$ with $a \neq 1$ coincides with the integration with respect to $w=a(a-1)^{-1} z^{a-1}$ followed by multiplication of $z^{-(a-1)}$, the non-integral power being understood to mean the principal branch. More precisely, we have

$$
\mathcal{L}(a) f(z)=\left\{\begin{array}{lr}
\frac{1}{z^{a-1}} \int_{\infty}^{w} F(\omega) d \omega & (0<a<1), \\
\frac{1}{z^{a-1}} \int_{0}^{w} F(\omega) d \omega & (a>1),
\end{array}\right.
$$

where $w=a(a-1)^{-1} z^{a-1}, F(w)=f\left(\left(a^{-1}(a-1) w\right)^{1 /(a-1)}\right)$ and the integration paths are 
the half straight line $\{\arg \omega=\pi-(1-a) \arg z, \infty>|\omega|>|w|\}$ for $0<a<1$ and the segment $\{\arg \omega=(a-1) \arg z, 0<|\omega|<|w|\}$ for $a>1$, respectively.

Proof. The operator $\mathcal{L}(a)$ is, by definition, given by

$$
\mathcal{L}(a) f(z)=a \int_{I} \frac{f(z t)}{t} t^{a-1} d t=\frac{a}{z^{a-1}} \int_{0}^{z} f(\zeta) \zeta^{a-2} d \zeta,
$$

the last integration being taken along the segment from 0 to $z$. We have only to change the variable of the last integral by $d \omega=a \zeta^{a-2} d \zeta$, or more concretely, by $\omega=a(a-1)^{-1} \zeta^{a-1}$. When $\zeta$ runs along the segment from 0 to $z, \omega$ runs along the respective integration path stated in the theorem.

It will be seen that the relation

$$
\frac{d}{d \log z} \mathcal{L}(a) f(z)=a f(z)-(a-1) \mathcal{L}(a) f(z)
$$

holds for any $a>0$. This may be regarded as a straightforward generalization of the already mentioned relation $(d / d \log z) \mathcal{L}(1) f(z)=f(z)$ corresponding to $a=1$. Here we state it in slightly general form:

THEOREM 5. For any $a>0$ and $\lambda \geqq 1$, we have

$$
\frac{d}{d \log z} \mathcal{L}(a)^{\lambda}=a \mathcal{L}(a)^{\lambda-1}-(a-1) \mathcal{L}(a)^{\lambda},
$$

$\mathcal{L}(a)^{0}$ being understood to be the identity operator.

Proof. By differentiating the defining equation of $\mathcal{L}(a)^{\lambda} f(z)$, we obtain

$$
\frac{d}{d \log z} \mathcal{L}(a)^{\lambda} f(z)=\frac{a^{\lambda}}{\Gamma(\lambda)} \int_{I} z f^{\prime}(z t) t^{a-1}\left(\log \frac{1}{t}\right)^{\lambda-1} d t
$$

which becomes after integration by parts

$$
\begin{aligned}
\frac{d}{d \log z} \mathcal{L}(a)^{\lambda} f(z)= & \frac{a^{\lambda}}{\Gamma(\lambda)}\left[f(z t) t^{a-1}\left(\log \frac{1}{t}\right)^{\lambda-1}\right]_{0}^{1} \\
& -\int_{I} f(z t)\left(-(\lambda-1) t^{a-2}\left(\log \frac{1}{t}\right)^{\lambda-2}+(a-1) t^{a-2}\left(\log \frac{1}{t}\right)^{\lambda-1}\right) d t .
\end{aligned}
$$

Here we remember $f \in \mathscr{I}$ and $a>0$. We get for $\lambda=1$

$$
\begin{aligned}
\frac{d}{d \log z} \mathcal{L}(a) f(z) & =a\left(f(z)-(a-1) \int_{I} f(z t) t^{a-2} d t\right) \\
& =a f(z)-(a-1) \mathcal{L}(a) f(z),
\end{aligned}
$$

while we obtain for $\lambda>1$ 


$$
\begin{aligned}
\frac{d}{d \log z} \mathcal{L}(a)^{\lambda} f(z)= & \frac{a^{\lambda}}{\Gamma(\lambda)}\left((\lambda-1) \int_{I} f(z t) t^{a-2}\left(\log \frac{1}{t}\right)^{\lambda-2} d t\right. \\
& \left.\quad-(a-1) \int_{I} f(z t) t^{a-2}\left(\log \frac{1}{t}\right)^{\lambda-1} d t\right) \\
= & a \mathcal{L}(a)^{\lambda-1} f(z)-(a-1) \mathcal{L}(a)^{\lambda} f(z) .
\end{aligned}
$$

In the following lines we shall consider the relation of $\mathcal{L}(a)$ to the ordinary integration operator $\mathcal{g}$ defined by

$$
\mathcal{I} f(z)=\int_{0}^{z} f(\zeta) d \zeta .
$$

For that purpose we attempt to derive the expression for $\mathcal{L}(a)$ in terms of $\mathcal{I}$ and its iterations. For the sake of brevity we make use of Pochhammer's symbol

$$
(x)_{n}=\frac{\Gamma(x+n)}{\Gamma(x)}=\prod_{j=0}^{n-1}(x+j) \quad(n=0,1, \cdots),
$$

the empty product denoting unity; in particular, $(x)_{0}=1$ even for $x=0$.

THEOREM 6. For any $a>0$ we have

$$
\mathcal{L}(a)=a \sum_{\kappa=1}^{\infty} \frac{(2-a)_{\kappa-1}}{z^{k}} g^{\kappa} .
$$

In particular, when $a=k>1$ is an integer, the right hand expression reduces to finite sum consisting of the beginning $k-1$ terms.

Proof. Since $|z-\zeta|<|z|$ holds on the integration path in the expression for $\mathcal{L}(a)$ except at $\zeta=0$, we have

$$
\begin{aligned}
\zeta^{a-2} & =z^{a-2}\left(1-\frac{z-\zeta}{z}\right)^{a-2} \\
& =z^{a-2} \sum_{\kappa=0}^{\infty}(-1)^{\kappa}\left(\begin{array}{c}
a-2 \\
\kappa
\end{array}\right)\left(\frac{z-\zeta}{z}\right)^{\kappa} \\
& =z^{a-2} \sum_{\kappa=1}^{\infty} \frac{(2-a)_{\kappa-1}}{(\kappa-1) !} \frac{1}{z^{\kappa-1}}(z-\zeta)^{\kappa-1}
\end{aligned}
$$

Substitution followed by termwise integration yields

$$
\begin{aligned}
\mathcal{L}(a) f(z) & =a \sum_{\kappa=1}^{\infty} \frac{(2-a)_{\kappa-1}}{z^{\kappa}} \frac{1}{(\kappa-1) !} \int_{0}^{z} f(\zeta)(z-\zeta)^{\kappa-1} d \zeta \\
& =a \sum_{\kappa=1}^{\infty} \frac{(2-a)_{\kappa-1}}{z^{\kappa}} g^{\kappa} f(z) .
\end{aligned}
$$

When $a=k>1$ is an integer, then $(2-k)_{k-1}$ vanishes for every $\kappa \geqq k$. - The 
case $a=1$ is exceptional in the sense that every term in the summand for $\mathcal{L}(1)$ does not vanish.

It may be noted that, for integral value of $a$, the relation in the theorem can also be inductively varified, by making use of integration by parts. On the other hand, it is remarked that the operator $\mathcal{L}(2)$ was observed by Libera [4] and Livingston [5] and that $\mathcal{L}(a)$ for integer $a>1$ was studied by Bernardi [1], both in connection with some classes of univalent functions.

\section{Generalization.}

By relaxing the restriction that the referring probability measure $\sigma$ is a monomial, we now consider a probability measure defined by a power series

$$
\sigma(t)=\sum_{k=1}^{\infty} \omega_{k} t^{k}
$$

with convergence radius greater than unity: $\lim \sup _{k \rightarrow \infty} \sqrt[k]{\left|\omega_{k}\right|}<1$. In view of the condition that $\sigma$ is a probability measure, we have to suppose

$$
\rho(t)=\sigma^{\prime}(t)=\sum_{k=1}^{\infty} k \omega_{k} t^{k-1} \geqq 0 \quad(t \in I), \quad \sigma(1)=\sum_{k=1}^{\infty} \omega_{k}=1 .
$$

THEOREM 7. Let $\sigma$ satisfy the just mentioned conditions. Then the operator $\mathcal{L}[\rho]$ defined by

$$
\mathcal{L}[\rho] f(z)=\int_{I} \frac{f(z t)}{t} \rho(t) d t \quad(f \in \mathscr{F})
$$

is represented in terms of the ordinary integration operator $g$ in the form

$$
\mathcal{L}[\rho]=\sum_{\kappa=1}^{\infty}(-1)^{\kappa-1} \frac{\varphi^{(\kappa-1)}(1)}{z^{\kappa}} g^{\kappa},
$$

where $\varphi$ is defined by

$$
\varphi(t)=\frac{\rho(t)}{t}=\sum_{k=1}^{\infty} k \omega_{k} t^{k-2}
$$

Proof. By substituting the expressions for $\mathcal{L}(k)(k=1,2, \cdots)$ derived in Theorem 6, we obtain

$$
\begin{aligned}
\mathcal{L}[\rho] & =\sum_{k=1}^{\infty} \omega_{k} \mathcal{L}(k) \\
& =\omega_{1} \sum_{\kappa=1}^{\infty} \frac{(\kappa-1) !}{z^{\kappa}} \mathcal{g}^{\kappa}+\sum_{k=2}^{\infty} \omega_{k} k \sum_{\kappa=1}^{k-1}(-1)^{\kappa-1} \frac{(k-2) !}{(k-\kappa-1) ! z^{\kappa}} g^{\kappa} \\
& =\sum_{\kappa=1}^{\infty} \frac{\Phi_{\kappa}}{z^{\kappa}} \mathcal{J}^{\kappa}, \quad \text { say. }
\end{aligned}
$$


The coefficients of the last expression are given by

$$
\begin{aligned}
\Phi_{\kappa} & =(\kappa-1) ! \omega_{1}+(-1)^{\kappa-1} \sum_{k=\kappa+1}^{\infty} k \frac{(k-2) !}{(k-\kappa-1) !} \omega_{k} \\
& =(-1)^{\kappa-1}\left[\frac{d^{\kappa-1}}{d t^{\kappa-1}}\left(\frac{\omega_{1}}{t}+\sum_{k=2}^{\infty} k \omega_{k} t^{k-2}\right)\right]^{t=1} \\
& =(-1)^{\kappa-1} \varphi^{(\kappa-1)}(1),
\end{aligned}
$$

and hence the desired result.

The result just derived can be more slightly generalized with respect to the referring measure $\sigma$.

THEOREM 8. Let a probability measure $\sigma$ be given by

$$
\sigma(t)=\int_{0}^{\infty} t^{a} d \tau(a)
$$

where a measure $\tau$ defined on $(0, \infty)$ satisfies the conditions

$$
\rho(t)=\sigma^{\prime}(t)=\int_{0}^{\infty} a t^{a-1} d \tau(a) \geqq 0 \quad(t \in I), \quad \sigma(1)=\int_{0}^{\infty} d \tau(a)=1 .
$$

Then we have

$$
\mathcal{L}[\rho]=\sum_{\kappa=1}^{\infty}(-1)^{\kappa-1} \frac{\varphi^{(\kappa-1)}(1)}{z^{\kappa}} g^{\kappa},
$$

where $\varphi$ is defined by

$$
\varphi(t)=\frac{\rho(t)}{t}=\int_{0}^{\infty} a t^{a-2} d \tau(a)
$$

Proof. The proof proceeds quite similar as for the previous theorem. In fact, we have

$$
\begin{aligned}
\mathcal{L}[\rho] f(z) & =\int_{I} \frac{f(z t)}{t} d \int_{0}^{\infty} t^{a} d \tau(a) \\
& =\int_{0}^{\infty} d \tau(a) \int_{I} \frac{f(z t)}{t} a t^{a-1} d t=\int_{0}^{\infty} \mathcal{L}(a) f(z) d \tau(a) .
\end{aligned}
$$

Hence, by substituting the expression for $\mathcal{L}(a)$ derived in Theorem 6 , we obtain

$$
\mathcal{L}[\rho]=\int_{0}^{\infty} a \sum_{\kappa=1}^{\infty} \frac{(2-a)_{\kappa-1}}{z^{\kappa}} \mathcal{g}^{\kappa} d \tau(a)=\sum_{\kappa=1}^{\infty} \frac{\Phi_{k}}{z^{\kappa}} \mathcal{g}^{k}, \quad \text { say . }
$$

The coefficients of the last expression are given by 


$$
\begin{aligned}
\Phi_{k} & =\int_{0}^{\infty} a(2-a)_{\kappa-1} d \tau(a) \\
& =(-1)^{\kappa-1}\left[\frac{d^{\kappa-1}}{d t^{\kappa-1}} \int_{0}^{\infty} a t^{a-2} d \tau(a)\right]^{t=1}=(-1)^{\kappa-1} \varphi^{(\kappa-1)}(1),
\end{aligned}
$$

and hence the result.

REMARK. Throughout this section the restriction $\rho(t)=\sigma^{\prime}(t) \geqq 0 \quad(t \in I)$ is really inessential, since the discussions concern to derive relations involving equality alone. From this standpoint we supplement here an example concerning Theorem 7.

We consider $\sigma^{\prime}$ (of indefinite sign) given by

$$
\sigma(t)=A_{2 m} \int_{0}^{t} \tau P_{2 m}(\tau) d \tau \quad(m \geqq 1),
$$

where $P_{2 m}$ denotes the Legendre polynomial of degree $2 m$ and $A_{2 m}$ is the normalization factor determined by $\sigma(1)=1$. By means of Rodrigues formula we get after repeated integration by parts

$$
\frac{1}{A_{2 m}}=\int_{I} \tau P_{2 m}(\tau) d \tau=\frac{(-1)^{m-1}}{2^{2 m}} \frac{(2 m-2) !}{(m-1) !(m+1) !} .
$$

By making use of a familiar formula

$$
P_{n}(t)=\sum_{\nu=0}^{n}(-1)^{\nu} \frac{(n+\nu) !}{\nu !^{2}(n-\nu) !}\left(\frac{1-t}{2}\right)^{\nu},
$$

we get after repeated differentiation

$$
P_{n}^{(\kappa-1)}(t)=\sum_{\nu=\kappa-1}^{n}(-1)^{\nu} \frac{(n+\nu) !}{\nu !^{2}(n-\nu) !}\left(-\frac{1}{2}\right)^{\kappa-1} \frac{\nu !}{(\nu-\kappa+1) !}\left(\frac{1-t}{2}\right)^{\nu-\kappa+1} .
$$

Thus, for $\varphi(t)=A_{2 m} P_{2 m}(t)$ we obtain the value of $\varphi^{(\kappa-1)}$ and finally

$$
\mathcal{L}\left[\sigma^{\prime}\right]=(-1)^{m-1} 2^{2 m} \frac{(m-1) !(m+1) !}{(2 m-2) !} \sum_{\kappa=1}^{2 m+2} \frac{(-1)^{\kappa-1} \cdot(2 m+\kappa-1) !}{(\kappa-1) !(2 m-\kappa+1) !} \frac{1}{2^{\kappa-1}} \frac{1}{z^{\kappa}} g^{\kappa} .
$$

For $m=0$, we have $P_{0}(\tau)=1, A_{0}=2 ; \sigma(t)=t^{2}$ and

$$
\mathcal{L}\left[\sigma^{\prime}\right]=\mathcal{L}(2)=\frac{2}{z} \mathcal{g},
$$

while for $m=1 / 2$, we have $P_{1}(\tau)=\tau, A_{1}=3 ; \sigma(t)=t^{3}$ and

$$
\mathcal{L}\left[\sigma^{\prime}\right]=\mathcal{L}(3)=3 \frac{1}{z} \mathcal{g}-\frac{1}{z^{2}} \mathcal{g}^{2} .
$$

However, the case with odd suffix greater than 1 has been rejected, since we would have $1 / A_{n}=0$ for any odd integer $n \geqq 3$. 
In this occasion we state another remark. The discussions developed in $\S 2$ and $\S 3$ for the case generated by

$$
\rho(t ; a)=a t^{a-1}, \quad \rho_{\lambda}(t ; a)=\frac{a^{\lambda}}{\Gamma(\lambda)} t^{a-1}\left(\log \frac{1}{t}\right)^{\lambda-1}
$$

will be generalized formally to the case

$$
\rho(t ; a, b)=\frac{a^{b}}{\Gamma(b)} t^{a-1}\left(\log \frac{1}{t}\right)^{b-1} .
$$

However, the latter can be reduced essentially to the former. In fact, we have only to take into account the relation

$$
\rho_{\lambda}(t ; a, b)=\rho_{b \lambda}(t ; a, 1)=\rho_{b \lambda}(t ; a) .
$$

\section{Hadamard product.}

The Hadamard product $*$ of two power series

$$
\varphi(z)=\sum_{\nu=1}^{\infty} a_{\nu} z^{\nu}, \quad \phi(z)=\sum_{\nu=1}^{\infty} b_{\nu} z^{\nu}
$$

is defined by

$$
\varphi * \psi(z)=\sum_{\nu=1}^{\infty} a_{\nu} b_{\nu} z^{\nu} .
$$

It is readily seen that $\varphi, \phi \in \mathscr{I}$ implies $\varphi * \psi \in \mathscr{F}$ and the particular function

$$
\chi(z)=\frac{z}{1-z}=\sum_{\nu=1}^{\infty} z^{\nu}
$$

plays the role of unit function with respect to the operation $*$ in the class $\mathscr{F}$; namely, $f * \chi=\chi * f=f(f \in \mathscr{T})$.

On the other hand, any operator $\mathcal{L}$ under consideration satisfies

$$
\begin{aligned}
\mathcal{L}(\varphi * \psi)(z) & =\int_{I} \frac{(\varphi * \psi)(z t)}{t} d \sigma(t)=\int_{I}\left(\varphi(z) * \frac{\phi(z t)}{t}\right) d \sigma(t) \\
& =\varphi(z) * \int_{I} \frac{\phi(z t)}{t} d \sigma(t)=(\varphi * \mathcal{L} \psi)(z),
\end{aligned}
$$

whence follows, in particular,

$$
\mathcal{L} f=\mathcal{L}(f * \chi)=f * \mathcal{L} \chi .
$$

Thus, the action of $\mathcal{L}$ on any function $f \in \mathscr{F}$ is reduced to the Hadamard product of $f$ with $\mathcal{L} \chi$.

If we consider, for instance, the operator $\mathcal{L}(a)$ defined in $\S 2$, we have an expansion of $\mathcal{L}(a) \chi$ in the form 


$$
\mathcal{L}(a) \chi(z)=a \sum_{\nu=1}^{\infty} \frac{z^{\nu}}{\Gamma(\nu+a)} .
$$

On the other hand, we have derived an expression for $\mathcal{L}(a)$ in terms of $\left\{g^{x}\right\}$, which, in particular, yields

$$
\sum_{\nu=1}^{\infty} \frac{z^{\nu}}{\Gamma(\nu+a)}=\sum_{\kappa=1}^{\infty} \frac{(2-a)_{\kappa-1}}{z^{\kappa}} g^{\kappa} \chi(z)
$$

valid for $a>0$. Now, as readily seen directly, $g^{\kappa} \chi$ is expressed by the expansion

$$
g^{\kappa} \chi(z)=z^{\kappa} \sum_{\nu=1}^{\infty} \frac{z^{\nu}}{(\nu+1)_{\kappa}} .
$$

By substituting this into the above relation and comparing the coefficients of $z^{\nu}$, we obtain an identity

$$
\frac{1}{\Gamma(\nu+a)}=\sum_{\kappa=1}^{\infty} \frac{(2-a)_{\kappa-1}}{(\nu+1)_{\kappa}} \quad(\nu=1,2, \cdots) .
$$

Now, $g^{\kappa} \chi(z)$ is for any integer $\kappa \geqq 0$ an elementary function. We have, for instance,

$$
\mathfrak{S} \chi(z)=\log \frac{1}{1-z}-z, \quad \mathfrak{g}^{2} \chi(z)=-(1-z) \log \frac{1}{1-z}+z-\frac{z^{2}}{2} .
$$

For any integer $\kappa \geqq 2$ we can derive similar explicit expression in the form

$$
\begin{aligned}
\mathcal{J}^{\kappa} \chi(z)= & \frac{(-1)^{\kappa-1}}{(\kappa-1) !}(1-z)^{\kappa-1} \log \frac{1}{1-z}+\frac{(-1)^{\kappa-1}}{\kappa !}(1-z)^{\kappa} \\
& +\frac{(-1)^{\kappa-1}}{(\kappa-1) !} \sum_{\jmath=2}^{\kappa-1} \frac{1}{\jmath} \cdot(1-z)^{\kappa-1}+\sum_{\jmath=0}^{\kappa-2}+\frac{(-1)^{\jmath}}{j !} \frac{1}{(\kappa-j) !(\kappa-\jmath-1)}(1-z)^{\jmath},
\end{aligned}
$$

the empty sum being to be understood zero. It is verified, for instance, by induction though the actual calculation is somewhat troublesome.

\section{Distortion inequalities.}

In the previous paper [2], we discussed some distortion properties on the family $\left\{\mathcal{L}^{\lambda}\right\}$ generated by a general measure $\sigma$, and specialized them in the case of $\left\{\mathcal{L}(1)^{\lambda}\right\}$. We supplement here these results by observing the family $\left\{\mathcal{L}(a)^{\lambda}\right\}$ with $a>0$. by

First, for a fixed pair $f, g \in \mathscr{I}$ we consider the quantities $M$ and $N$ defined

$$
\begin{gathered}
M(r ; a, \lambda, \mu)=\max _{|z|=r}\left|\mathcal{L}(a)^{\lambda} f(z)-\mathcal{L}(a)^{\mu} g(z)\right|, \\
N(r ; a, \lambda)=\max _{|z|=r}\left|\mathcal{L}(a)^{\lambda} f(z)-z\right| .
\end{gathered}
$$

THEOREM 9. For any $f, g \in \mathscr{F}$ the quantity $M(r ; a, \lambda+\delta, \mu+\delta)$ decreases with 
respect to $\delta \geqq 0$. More precisely, for $\delta^{\prime}>\delta \geqq 0$ we have

$$
\left(\frac{a+1}{a}\right)^{\delta^{\prime}} M\left(r ; a, \lambda+\delta^{\prime}, \mu+\delta^{\prime}\right) \leqq\left(\frac{a+1}{a}\right)^{\delta} M(r ; a, \lambda+\delta, \mu+\delta) .
$$

Proof. As shown in [2], we have

$$
M(r ; a, \lambda+\delta, \mu+\delta) \leqq M(r ; a, \lambda, \mu) \int_{I} t d \sigma_{\delta}(t ; a) .
$$

The last factor of the right hand member is in the present case equal to

$$
\int_{I} t d \sigma_{\delta}(t ; a)=\frac{a^{\delta}}{\Gamma(\delta)} \int_{I} t^{a}\left(\log \frac{1}{t}\right)^{\delta-1} d t=\left(\frac{a}{a+1}\right)^{\delta} .
$$

Let $0 \leqq \delta<\delta^{\prime}$. Then, by replacing $\lambda, \mu$ and $\delta$ in the above inequality by $\lambda+\delta$, $\mu+\delta$ and $\delta^{\prime}-\delta$, respectively, we obtain the desired result.

COROLLARY. For any $f \in \mathscr{F}$ the quantity $((a+1) / a)^{\delta} N(r ; a, \lambda+\delta)$ decreases with respect to $\delta \geqq 0$.

Proof. Since $L(a)^{\mu} z$ becomes $z$ for any $\mu$, the quantity $M(r ; a, \lambda, \mu)$ reduces to $N(r ; a, \lambda)$ provided $g(z)=z$. Hence, the assertion follows from the theorem by only substituting $g(z)=z$.

By the way, it follows from the Corollary that

$$
\left(\frac{a+1}{a}\right)^{\delta} N(r ; a, \lambda+\delta) \leqq N(r ; a, \lambda) \text {. }
$$

If we replace here both $\lambda$ and $\delta$ by $\lambda / 2$, we get

$$
N(r ; a, \lambda) \leqq\left(\frac{a}{a+1}\right)^{\lambda / 2} N\left(r ; a, \frac{\lambda}{2}\right) .
$$

In view of this inequality, we see that the first limit relation stated in Theorem 3 is again verified.

Next, for a fixed $f \in \mathscr{I}$ we observe the quantities $h$ and $H$ defined by

$$
\begin{aligned}
& h_{\lambda}(r ; a) \\
& H_{\lambda}(r ; a)=\max _{|z|=r}^{\min } \operatorname{Re} \frac{\mathcal{L}(a)^{\lambda} f(z)}{z} .
\end{aligned}
$$

THEOREM 10. For any $f \in \mathscr{F}$ and $\delta>0$ we have

$$
\begin{aligned}
& h_{\lambda+\delta}(r ; a) \geqq h_{\lambda}(r ; a)+\Phi(\delta, a)\left(1-h_{\lambda}(r ; a)\right), \\
& H_{\lambda+\delta}(r ; a) \leqq H_{\lambda}(r ; a)-\Phi(\delta, a)\left(H_{\lambda}(r ; a)-1\right)
\end{aligned}
$$

where $\Phi$ is given by 


$$
\Phi(\delta, a)=1-2 a^{\delta} \sum_{\nu=2}^{\infty} \frac{(-1)^{\nu}}{(\nu+a-1)^{\delta}} .
$$

The equality sign in either estimation does not appear for any $r \in(0,1)$ unless $f(z)$ $=z$. If , in particular, $a=k$ is a positive integer, $\Phi(\delta, k)$ is expressible in the form

$$
\Phi(\delta, k)=1+2(-1)^{k-1} k^{\delta}\left(\left(1-2^{1-\delta}\right) \zeta(\delta)+\sum_{\kappa=1}^{k} \frac{(-1)^{\kappa-1}}{\kappa^{\delta}}\right),
$$

$\zeta$ denoting Riemann zeta function.

Proof. The inequalities having been generally shown in [2], it suffices to verify the expression for $\Phi$. We first have

$$
\begin{aligned}
\Phi(\delta, a) & =\int_{I} \frac{1-t}{1+t} d \sigma_{\delta}(t ; a) \\
& =\frac{a^{\delta}}{\Gamma(\delta)} \int_{I} \frac{1-t}{1+t} t^{a-1}\left(\log \frac{1}{t}\right)^{\delta-1} d t \\
& =\frac{a^{\delta}}{\Gamma(\delta)} \int_{I}\left(1-2 \sum_{\nu=2}^{\infty}(-1)^{\nu} t^{\nu-1}\right) t^{a-1}\left(\log \frac{1}{t}\right)^{\delta-1} d t \\
& =1-2 a^{\delta} \sum_{\nu=2}^{\infty} \frac{(-1)^{\nu}}{(\nu+a-1)^{\delta}} .
\end{aligned}
$$

Next, in view of the formula

$$
\sum_{\kappa=1}^{\infty} \frac{(-1)^{\kappa-1}}{\kappa^{\delta}}=\left(1-2^{1-\delta}\right) \zeta(\delta),
$$

we get for a positive integer $k$ the relation

$$
\begin{aligned}
\sum_{\nu=2}^{\infty} \frac{(-1)^{\nu}}{(\nu+k-1)^{\delta}} & =\sum_{\kappa=k+1}^{\infty} \frac{(-1)^{\kappa-k+1}}{\kappa^{\delta}}=(-1)^{k}\left(\sum_{k=1}^{\infty}-\sum_{k=1}^{k}\right) \frac{(-1)^{\kappa-1}}{\kappa^{\delta}} \\
& =(-1)^{k}\left(1-2^{1-\delta}\right) \zeta(\delta)+(-1)^{k} \sum_{\kappa=1}^{k} \frac{(-1)^{\kappa}}{\kappa^{\delta}} .
\end{aligned}
$$

By substituting this in the above expression for $\Phi(\delta, a)$ with $a=k$, we obtain its desired expression.

COROLLARY. We have

$$
\begin{aligned}
& h_{\lambda+\delta}(r ; a) \geqq h_{\lambda}(r ; a)+\left(1-e^{-\Phi^{\prime}(0, a) \delta}\right)\left(1-h_{\lambda}(r ; a)\right), \\
& H_{\lambda+\delta}(r ; a) \leqq H_{\lambda}(r ; a)-\left(1-e^{-\Phi^{\prime}(0, a) \delta}\right)\left(H_{\lambda}(r ; a)-1\right),
\end{aligned}
$$

where $\Phi^{\prime}$ is given by 


$$
\begin{aligned}
\Phi^{\prime}(0, a) & =\left[\frac{\partial}{\partial \delta} \Phi(\delta, a)\right]^{\delta=+0} \\
& =2 \lim _{\delta \rightarrow+0} \sum_{\nu=2}^{\infty}(-1)^{\nu} \frac{\log (\nu+a-1)-\log a}{(\nu+a-1)^{\delta}} .
\end{aligned}
$$

If, in particular, $a=k$ is a positive integer, then

$$
\Phi^{\prime}(0, k)=2(-1)^{k-1} \log \frac{k ! \sqrt{\pi / 2}}{\left(2^{[k / 2]} \cdot[k / 2] !\right)^{2}}-\log k .
$$

Proof. We first note that $\Phi(+0, a)=0$. In fact, by means of integration by parts, we get

$$
\begin{aligned}
\Phi(\delta, a) & =\frac{a^{\delta}}{\Gamma(\delta)} \int_{I} \frac{1-t}{1+t} t^{a-1}\left(\log \frac{1}{t}\right)^{\delta-1} d t \\
& =\frac{a^{\delta}}{\Gamma(\delta+1)}\left\{\left[-\frac{1-t}{1+t} t^{a}\left(\log \frac{1}{t}\right)^{\delta}\right]_{0}^{1}+\int_{I} \frac{d}{d t}\left(\frac{1-t}{1+t} t^{a}\right) \cdot\left(\log \frac{1}{t}\right)^{\delta} d t\right\} \\
& =\frac{a^{\delta}}{\Gamma(\delta+1)} \int_{I} \frac{d}{d t}\left(\frac{1-t}{1+t} t^{a}\right) \cdot\left(\log \frac{1}{t}\right)^{\delta} d t
\end{aligned}
$$

whence readily follows

$$
\Phi(+0, a)=\int_{I} \frac{d}{d t}\left(\frac{1-t}{1+t} t^{a}\right) d t=0 .
$$

The first inequality in the theorem yields

$$
\frac{h_{\lambda+\delta}(r ; a)-h_{\lambda}(r ; a)}{\delta} \geqq \frac{\Phi(\delta, a)}{\delta}\left(1-h_{\lambda}(r ; a)\right),
$$

whence follows, as $\delta$ tends to +0 , the inequality

$$
\frac{\partial}{\partial \lambda} h_{\lambda}(r ; a) \geqq \Phi^{\prime}(0, a)\left(1-h_{\lambda}(r ; a)\right) .
$$

This linear differential inequality can be brought readily into finite form. In fact, by rewriting it in the form

$$
\frac{\partial}{\partial \lambda}\left(e^{\Phi^{\prime}(0, a) \lambda} h_{\lambda}(r ; a)\right) \geqq \Phi^{\prime}(0, a) e^{\Phi^{\prime}(0, a) \lambda}
$$

and then integrating with respect to $\lambda$ over the interval $(\lambda, \lambda+\delta)$, we obtain the desired estimation for $h$. Similar argument applies also for $H$. Next, we have in view of the expression for $\Phi(\delta, a)$ given in the theorem

$$
\frac{\partial}{\partial \delta} \Phi(\delta, a)=2 a^{\delta} \sum_{\nu=2}^{\infty}(-1)^{\nu} \frac{\log (\nu+a-1)-\log a}{(\nu+a-1)^{\delta}},
$$

whence follows the desired expression for $\Phi^{\prime}(0, a)$. Finally, if $a=k$ is a positive integer, we see that 


$$
\begin{aligned}
\frac{\partial}{\partial \delta} \Phi(\delta, k)= & 2(-1)^{k-1} k^{\delta} \log k\left(\left(1-2^{1-\delta}\right) \zeta(\delta)-\sum_{\kappa=1}^{k} \frac{(-1)^{\kappa-1}}{\kappa^{\delta}}\right) \\
& +2(-1)^{k-1} k^{\delta}\left(\left(1-2^{1-\delta}\right) \zeta^{\prime}(\delta)+2^{1-\delta} \log 2 \cdot \zeta(\delta)+\sum_{\kappa=2}^{k} \frac{(-1)^{\kappa-1}}{\kappa^{\delta}} \log \kappa\right) .
\end{aligned}
$$

In view of $\zeta(0)=-1 / 2$ and $\zeta^{\prime}(0)=-(1 / 2) \log 2 \pi$, we get

$$
\begin{aligned}
\Phi^{\prime}(0, k)= & 2(-1)^{k-1} \log k\left(\frac{1}{2}-\sum_{\kappa=1}^{k}(-1)^{\kappa-1}\right) \\
& +2(-1)^{k-1}\left(\frac{1}{2} \log 2 \pi-\log 2+\sum_{k=2}^{k}(-1)^{\kappa-1} \log \kappa\right),
\end{aligned}
$$

which becomes the desired form, by remembering the elementary relations

$$
\frac{1}{2}-\sum_{k=1}^{k}(-1)^{\kappa-1}=\frac{(-1)^{k}}{2}, \quad \sum_{k=2}^{k}(-1)^{\kappa-1} \log \kappa=\log \frac{k}{\left(2^{[k / 2]} \cdot[k / 2] !\right)^{2}} .
$$

In the following lines, we shall supplement some properties of the quantities $\Phi(\delta, a)$ and $\Phi^{\prime}(0, a)$ contained in Theorem 10 and its Corollary.

For lower values of $\delta, a$ we see that

$$
\Phi(1,1)=2 \log 2, \quad \Phi(2,1)=\frac{\pi^{2}}{6}, \quad \Phi(1,2)=3-4 \log 2
$$

and hence, in particular, $\Phi(2,1)>\Phi(1,1)>\Phi(1,2)$. Now, we shall indicate that $\Phi(\delta, a)$ shows such monotoneity in general.

THEOREM 11. For any fixed $a>0$ we have

$$
\Phi(+0, a)=0 \quad \text { and } \quad \Phi(\infty, a)=1 .
$$

When $\delta$ increases from 0 to $\infty, \Phi(\delta, a)$ increases structly from 0 to 1.

Proof. The relation $\Phi(+0, a)=0$ has been shown on the way of proving the Corollary of Theorem 10. Next, we have

$$
1-\Phi(\delta, a)=\frac{a^{\delta}}{\Gamma(\delta)} \int_{I} \frac{2 t}{1+t} t^{a-1}\left(\log \frac{1}{t}\right)^{\delta-1} d t>0 .
$$

Let any small positive number $\varepsilon$ be given. Then, $2 t /(1+t)<\varepsilon / 2$ as $t<\varepsilon / 4$ and hence

$$
\begin{aligned}
1-\Phi(\delta, a) & <\frac{a^{\delta}}{\Gamma(\delta)}\left(\frac{\varepsilon}{2} \int_{0}^{\varepsilon / 4}+\int_{\varepsilon / 4}^{1}\right) t^{a-1}\left(\log \frac{1}{t}\right)^{\delta-1} d t \\
& <\frac{a^{\delta}}{\Gamma(\delta)}\left(\frac{\varepsilon}{2} \int_{I} t^{a-1}\left(\log \frac{1}{t}\right)^{\delta-1} d t+\left(\log \frac{4}{\varepsilon}\right)^{\delta-1} \int_{I}^{t^{a-1}} d t\right)
\end{aligned}
$$




$$
=\frac{\varepsilon}{2}+\frac{1}{\Gamma(\delta)}\left(a \log \frac{4}{\varepsilon}\right)^{\delta-1} .
$$

In view of Stirling formula applied to $\Gamma(\delta)$, we see that

$$
\frac{1}{\Gamma(\delta)}\left(a \log \frac{4}{\varepsilon}\right)^{\delta-1} \sim \frac{1}{\sqrt{2 \pi \delta} e}\left(\frac{e a}{\delta} \log \frac{4}{\varepsilon}\right)^{\delta-1} \rightarrow 0 \quad \text { as } \delta \rightarrow \infty
$$

and hence there exists $\Delta(\varepsilon)$ such that $1-\Phi(\delta, a)<\varepsilon$ as $\delta>\Delta(\varepsilon)$. This shows $\Phi(\infty, a)=1$. Finally, let $0<\delta<\delta^{\prime}$. Then

$$
\Phi\left(\delta^{\prime}, a\right)-\Phi(\delta, a)=\int_{I} \frac{1-t}{1+t} t^{a-1}\left(\frac{a^{\delta^{\prime}}}{\Gamma\left(\delta^{\prime}\right)}\left(\log \frac{1}{t}\right)^{\delta^{\prime}-1}-\frac{a^{\delta}}{\Gamma(\delta)}\left(\log \frac{1}{t}\right)^{\delta-1}\right) d t .
$$

Put $T=\exp \left(-(1 / a)\left(\Gamma\left(\delta^{\prime}\right) / \Gamma(\delta)\right)^{1 /\left(\delta^{\prime}-\delta\right)}\right)$. Then we see that as $t \lessgtr T$

$$
\frac{a^{\delta^{\prime}}}{\Gamma\left(\delta^{\prime}\right)}\left(\log \frac{1}{t}\right)^{\delta^{\prime-1}} \gtrless \frac{a^{\delta}}{\Gamma(\delta)}\left(\log \frac{1}{t}\right)^{\delta-1} \text { and } \frac{1-t}{1+t} \gtrless \frac{1-T}{1+T}
$$

and hence

$$
\begin{aligned}
& \Phi\left(\delta^{\prime}, a\right)-\Phi(\delta, a) \\
> & \frac{1-T}{1+T} \int_{I} t^{a-1}\left(\frac{a^{\delta^{\prime}}}{\Gamma\left(\delta^{\prime}\right)}\left(\log \frac{1}{t}\right)^{\delta^{\prime}-1}-\frac{a^{\delta}}{\Gamma(\delta)}\left(\log \frac{1}{t}\right)^{\delta-1}\right) d t=0 .
\end{aligned}
$$

THEOREM 12. For any fixed $\delta>0$ we have

$$
\Phi(\delta,+0)=1 \quad \text { and } \quad \Phi(\delta, \infty)=0 .
$$

When a increases from 0 to $\infty, \Phi(\delta, a)$ decreases strictly from 1 to 0 .

Proof. We see that

$$
\begin{aligned}
\Phi(\delta, a) & =\frac{a^{\delta}}{\Gamma(\delta)} \int_{I}\left(t^{a-1}-\frac{2 t^{a}}{1+t}\right)\left(\log \frac{1}{t}\right)^{\delta-1} d t \\
& =1-\frac{2 a^{\delta}}{\Gamma(\delta)} \int_{I} \frac{t^{a}}{1+t}\left(\log \frac{1}{t}\right)^{\delta-1} d t \rightarrow 1 \quad \text { as } a \rightarrow+0,
\end{aligned}
$$

since the last integral remains finite for $\delta>0$. Or, the result could be derived more simply by means of the series form of $\Phi$. Next, let any small positive number $\varepsilon$ be given. Then, $(1-t) /(1+t)<\varepsilon / 2$ as $1>t>\eta \equiv(2-\varepsilon) /(2+\varepsilon)$ and hence

$$
\begin{aligned}
0<\Phi(\delta, a) & <\frac{a^{\delta}}{\Gamma(\delta)}\left(\int_{0}^{\eta}+\frac{\varepsilon}{2} \int_{\eta}^{1}\right) t^{a-1}\left(\log \frac{1}{t}\right)^{\delta-1} d t \\
& <\frac{a^{\delta}}{\Gamma(\delta)} \int_{0}^{\eta} t^{a-1}\left(\log \frac{1}{t}\right)^{\delta-1} d t+\frac{\varepsilon}{2} .
\end{aligned}
$$

Since $a^{\delta} t^{a-1} \rightarrow 0$ as $a \rightarrow \infty$ uniformly for $t \in[0, \eta]$, there exists $A(\varepsilon)$ such that $0<\Phi(\delta, a)<\varepsilon$ as $a>A(\varepsilon)$. Finally, let $0<a<a^{\prime}<1$. Then 


$$
\Phi\left(\delta, a^{\prime}\right)-\Phi(\delta, a)=\frac{1}{\Gamma(\delta)} \int_{I} \frac{1-t}{1+t}\left(a^{\prime \delta} t^{a^{\prime}-1}-a^{\delta} t^{a-1}\right)\left(\log \frac{1}{t}\right)^{\delta-1} d t
$$

We see that as $t \lessgtr\left(a / a^{\prime}\right)^{\delta /\left(a^{\prime}-a\right)}$

$$
\frac{1-t}{1+t} \gtrless \frac{1-\left(a / a^{\prime}\right)^{\delta /\left(a^{\prime}-a\right)}}{1+\left(a / a^{\prime}\right)^{\delta /\left(a^{\prime}-a\right)}} \quad \text { and } \quad a^{\prime \delta} t^{a^{\prime}-1} \lessgtr a^{\delta} t^{a-1}
$$

and hence

$$
\begin{aligned}
& \Phi\left(\delta, a^{\prime}\right)-\Phi(\delta, a) \\
< & \frac{1-\left(a / a^{\prime}\right)^{\delta /\left(a^{\prime}-a\right)}}{1+\left(a / a^{\prime}\right)^{\delta /\left(a^{\prime}-a\right)}} \frac{1}{\Gamma(\delta)} \int_{I}\left(a^{\prime \delta} t^{a^{\prime}-1}-a^{\delta} t^{a-1}\right)\left(\log \frac{1}{t}\right)^{\delta-1} d t=0 .
\end{aligned}
$$

REMARK. If $\phi(t)$ is a measurable function bounded on $I$ and left-continuous at 1 , a similar argument as above for deriving $\Phi(\delta, \infty)=0$ in which $(1-t) /(1+t)$ is replaced by $\phi(t)-\phi(1)$ yields

$$
\int_{I}(\phi(t)-\phi(1)) \rho_{\delta}(t ; a) d t \rightarrow 0, \quad \text { i. e., } \int_{I} \phi(t) \rho_{\delta}(t ; a) d t \rightarrow \phi(1) \quad \text { as } a \rightarrow \infty \text {. }
$$

This relation corresponds to the fact that the probability density

$$
\rho_{\delta}(t ; a)=\frac{a^{\delta}}{\Gamma(\delta)} t^{a-1}\left(\log \frac{1}{t}\right)^{\delta-1}
$$

is a kernel of singular integral tending to concentrate at $t=1$ as $a \rightarrow \infty$.

Now, we shall denote $\Phi^{\prime}(0, a)$ briefly by $\Psi(a)$, namely

$$
\Psi(a)=\Phi^{\prime}(0, a)=\left[\frac{\partial}{\partial \delta} \Phi(\delta, a)\right]^{\delta=+0}
$$

As shown in the Corollary of Theorem 10, the quantity $\Psi(k)$ with a positive integer $k$ is represented in terms of elementary expressions; in particular, we have

$$
\begin{aligned}
& \Psi(1)=\log \frac{\pi}{2}, \quad \Psi(2)=\log \frac{4}{\pi}, \quad \Psi(3)=\log \frac{3 \pi}{8}, \\
& \Psi(4)=\log \frac{32}{9 \pi}, \quad \Psi(5)=\log \frac{45 \pi}{128}, \quad \Psi(6)=\log \frac{256}{75 \pi}, \quad \text { etc. }
\end{aligned}
$$

We supplement here the monotoneity of $\Psi(a)$.

THEOREM 13. We have

$$
\Psi(+0)=\infty \quad \text { and } \quad \Psi(\infty)=0 .
$$

When a increases from 0 to $\infty, \Psi(a)$ decreases stractly from $\infty$ to 0 . 
Proof. The expression for $\Phi(\delta, a)$ obtained in the proof of the Corollary of Theorem 10 yields, after differentiation with respect to $\delta$,

$$
\begin{aligned}
\frac{\partial}{\partial \delta} \Phi(\delta, a)= & a^{\delta}\left(\frac{\log a}{\Gamma(\delta+1)}-\frac{\Gamma^{\prime}(\delta+1)}{\Gamma(\delta+1)^{2}}\right) \int_{I} \frac{d}{d t}\left(\frac{1-t}{1+t} t^{a}\right) \cdot\left(\log \frac{1}{t}\right)^{\delta} d t \\
& +\frac{a^{\delta}}{\Gamma(\delta+1)} \int_{I} \frac{d}{d t}\left(\frac{1-t}{1+t} t^{a}\right) \cdot\left(\log \frac{1}{t}\right)^{\delta} \log \log \frac{1}{t} d t,
\end{aligned}
$$

whence follows after integration by parts

$$
\Psi(a)=\int_{I} \frac{d}{d t}\left(\frac{1-t}{1+t} t^{a}\right) \cdot \log \log \frac{1}{t} d t=\int_{I} \frac{1-t}{1+t} t^{a-1}\left(\log \frac{1}{t}\right)^{-1} d t .
$$

The decresing property of $\Psi(a)$ is evident in view of the last expression. Now, for any $\varepsilon \in(0,1 / 2)$ we get

$$
\Psi(a)>\frac{1}{3} \varepsilon^{a} \int_{\varepsilon}^{1 / 2} t^{-1}\left(\log \frac{1}{t}\right)^{-1} d t=\frac{1}{3} \varepsilon^{a}\left[-\log \log \frac{1}{t}\right]_{\varepsilon}^{1 / 2},
$$

whence follows

$$
\liminf _{a \rightarrow+0} \Psi(a) \geqq \frac{1}{3}\left[-\log \log \frac{1}{t}\right]_{\varepsilon}^{1 / 2} .
$$

Since $\varepsilon \in(0,1 / 2)$ is arbitrary, we conclude $\Psi(+0)=\infty$. Next, since the integrand of the above integral expressing $\Psi(a)$ is uniformly bounded on $I$ for $a \geqq 2$ and tends to 0 as $a \rightarrow \infty$, it follows that $\Psi(\infty)=0$.

\section{REFERENCES}

[1] S. D. Bernardi, Convex and starlike univalent functions, Trans. Amer. Math. Soc., 135 (1969), 429-446.

[2] Y. Komatu, On a one-parameter additive family of operators defined on analytic functions regular in the unit disk, Bull. Fac. Sci.-Eng., Chuo Univ., 22 (1979), $1-22$.

[3] Y. Komatu, A one-parameter family of operators defined on analytic functions in a circle, Lecture Notes in Math. No. 768, Proc. Anal. Func./Kozubnik 1979, ed. by J. Ławrynowicz, Springer (1980), 292-300.

[4] R. J. Libera, Some classes of regular univalent functions, Proc. Amer. Math. Soc., 16 (1965), 755-758.

[5] A. E. Livingston, On the radius of univalence of certain analytic functions, Proc. Amer. Math. Soc., 17 (1966), 352-357. 